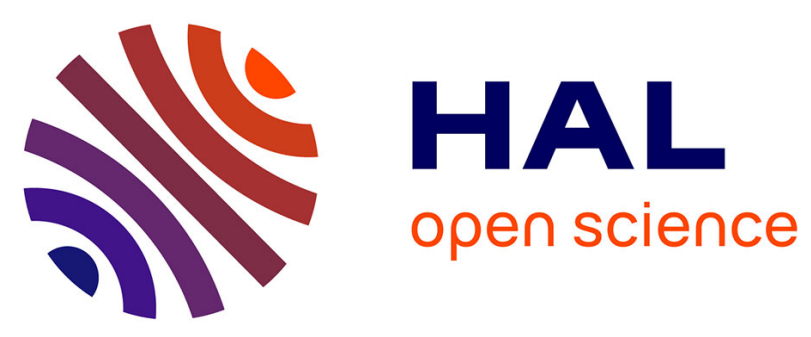

\title{
Le réseau local tout optique
}

\author{
R. Madigou, J. Le Rouzic
}

\section{- To cite this version:}

R. Madigou, J. Le Rouzic. Le réseau local tout optique. Revue de Physique Appliquée, 1984, 19 (2), pp.109-111. 10.1051/rphysap:01984001902010900 . jpa-00245160

\section{HAL Id: jpa-00245160 https://hal.science/jpa-00245160}

Submitted on 1 Jan 1984

HAL is a multi-disciplinary open access archive for the deposit and dissemination of scientific research documents, whether they are published or not. The documents may come from teaching and research institutions in France or abroad, or from public or private research centers.
L'archive ouverte pluridisciplinaire HAL, est destinée au dépôt et à la diffusion de documents scientifiques de niveau recherche, publiés ou non, émanant des établissements d'enseignement et de recherche français ou étrangers, des laboratoires publics ou privés. 
Classification

Physics Abstracts

$42.80-42.80 \mathrm{~S}$

\title{
Le réseau local tout optique
}

\author{
R. Madigou et J. Le Rouzic \\ Centre National d'Etudes des Télécommunications, LAB/ROC/TAC, B.P. 40, 22301 Lannion, France
}

(Reçu le 3 juin 1983, révisé et accepté le 29 août 1983)

\begin{abstract}
Résumé. - A partir de la description d'une maquette expérimentale, nous proposons une architecture de Réseau Local Tout Optique utilisant un maximum de technologies optiques comme la commutation spatiale à miroirs et un multiplexage à trois longueurs d'onde lumineuse.
\end{abstract}

Abstract. - Through the description of an experimental model, this paper proposes an all optical local network architecture using a maximum of optical technologies as a spatial switching through mirrors and a three wavelength-division-multiplex.

\section{Introduction.}

Les prochaines années connaîtront l'introduction massive de l'informatique, de la vidéo ou encore du son diffusé au domicile des abonnés grand public. En conséquence, de nouveaux réseaux verront le jour afin de véhiculer et de traiter des informations très diverses qu'elles soient analogiques ou numériques, faible et large bandes, uni- et bi-directionnelles... Ces réseaux devront satisfaire aux impératifs d'évolution tant sur le plan technique que sur celui des services dont le caractère interactif sera très dévelop̀pé. Visant ces objectifs, nous avons défini une architecture de "Réseau Local de Vidéocommunication Tout Optique " basée sur l'utilisation de la fibre optique et concrétisée par la maquette expérimentale présentée ci-après.

\section{Le réseau local tout optique.}

2.1 Caractéristiques Principales. - Le Réseau Local Tout Optique, à structure étoilée [1-2े], se compose principalement des sous-ensembles suivants : d'une part d'un autocommutateur siège de connexions optiques bidirectionnelles large bande et, d'autre part, de concentrateurs-distributeurs optiques [3] assurant conjointement la concentration de lignes d'abonnés sur des circuits d'accès à l'autocommutateur et la diffusion d'images animées (Télédistribution) vers ces mêmes abonnés. Par ailleurs, le concentrateur-distributeur interface les réseaux large bande et les réseaux à bas débit tels les réseaux de données, téléphonique, de signalisation, ..., ce qui conduit, par voie de conséquence, à un réseau de type multiservices dans la mesure où depuis l'usager jusqu'au concentrateur de rattachement le même support (fibre) sert pour la transmission des signaux relatifs à tous les services offerts. La vocation " tout optique " de ce réseau se traduit par l'introduction de la commutation optique [4] et d'un multiplexage à trois longueurs d'onde permettant ainsi l'attribution d'ine seule fibre optique (multimodale) par abonné [5].

\subsection{Descriptif De La maquette De RéSEAu Local} Tout OptiQue (Fig. 1).

2.2.1 Principe. - Modèle réduit d'un réseau de vidéocommunication, cette maquette, organisée autour d'une matrice de commutation spatiale optique $4 \times 4$

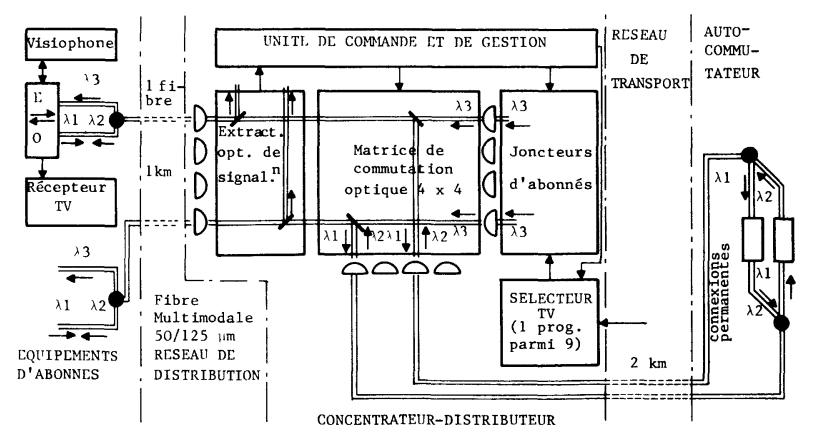

Fig. 1. - Maquette de réseau local tout optique.

[Working-Prototype of the all optical local network.] 
à miroirs, autorise le raccordement de quatre usagers lesquels ont accès à deux services de base : un service de type conversationnel large bande (Service visiophonique) et un service de type diffusé (un programme TV parmi neuf au moyen de deux magnétoscopes).

Ces usagers sont raccordés à la matrice par le biais d'une seule fibre optique $50 / 125 \mu \mathrm{m}$, chacun d'entre eux disposant d'un terminal visiophonique, d'un récepteur de télévision et d'une régie d'abonné assurant l'interface opto-électronique avec le réseau. $\mathrm{La}$ matrice optique $4 \times 4$ permet la concentration des fibres d'abonné pour le service commuté large bande et sert de point d'accès pour les signaux diffusés qui sont injectés au moyen d'émetteurs optiques individualisés. La concentration s'opère par déviation d'un faisceau lumineux (produit par un collimateur optique) à l'aide d'un miroir à couches multidiélectriques placé sur le trajet libre du faisceau. A l'entrée de la matrice, une très faible proportion du signal optique provenant de chaque fibre d'abonné est défléchie (miroir fixe à très faible coefficient de réflexion) de manière à pouvoir extraire le canal basse fréquence de signalisation d'abonné. Ce type de dispositif optique passif pourrait être utilisé pour interfacer les réseaux de types large bande et bas débit.

Après analyse de la signalisation émise par l'abonné, l'unité de commande et de gestion (à structure microprogrammée) peut diriger le reste du signal optique ( $95 \%$ environ du signal incident véhiculant les informations visiophoniques) vers le correspondant demandé par activation du miroir de commutation approprié, ou peut encore commander l'émission d'un programme TV si une diffusion a été sollicitée.

Les signaux diffusés traversent les miroirs sans perturbation en raison des caractéristiques de ces derniers qui sont réfléchissants vis-à-vis des signaux commutés (fenêtre optique $0,85 \mu \mathrm{m}$ ) et transparents aux signaux diffusés (fenêtre $1,3 \mu \mathrm{m}$ ), ce qui rend possible la simultanéité des deux services mis en ouvre.

Le temps de réponse du point de connexion optique (miroir + dispositif électromagnétique bistable) est de $15 \mathrm{~ms}$ pour une consommation de $30 \mathrm{~mJ}$ (uniquement à la commande), ce qui défie sur ce point tous les systèmes électroniques concurrents. Les pertes d'insertion sont de 3 à $4 \mathrm{~dB}$ environ avec des optiques simples et non traitées (cf. Tableau I).

Par ailleurs, ce type de commutation optique n'induit aucune limitation en bande passante électrique des signaux transmis et est totalement indépendant du type de ces signaux (analogiques ou numériques par exemple).

2.2.2 Multiplexage optique. Plan de fréquence. Modulation. - Le mode de transmission utilisé associe un multiplexage optique à trois longueurs d'onde $(0,79-$ $0,82-1,3 \mu \mathrm{m})$ et un multiplexage électrique sur les longueurs d'onde 0,79 et $1,3 \mu \mathrm{m}$ afin d'introduire en plus des signaux vidéo, les canaux de signalisation indispensables pour l'établissement des différents échanges.

Les dispositifs de multiplexage optique (cf. Tableau II) ont été réalisés par la S.A.T. (France) avec les éléments constitutifs Filtres Fabry-Pérot fournis par le laboratoire d'optique (Centre d'Etudes des couches minces) de l'ENSPM dans le cadre d'un marché d'étude. La modulation est du type analogique (FSK pour la signalisation dans une bande de $35 \mathrm{kHz}$ et PFM pour les signaux vidéo et audio-associés dans une bande de $15 . \mathrm{MHz}$ ).

Tableau II. - Le multiplexeur-démultiplexeur optique.

\section{[WDM device.]}

\begin{tabular}{|c|c|c|}
\hline \multirow{4}{*}{ CARACTERISTIQUES } & ORIGINE & Société SAT (France) \\
\hline & LONGUEURS D'ONDE: & $\begin{array}{l:ll}\lambda 1 & 0.79 \mu \mathrm{m} \\
\lambda 2 & : 0.82 \mu \mathrm{m} \\
\lambda 3 & : 1.3 \mu \mathrm{m}\end{array}$ \\
\hline & $\begin{array}{c}\text { SLPARATION SPECTRALE } \\
(\lambda 1, \lambda 2) / \lambda 3\end{array}$ & $\begin{array}{c}\text { FILTRE A COUCHES } \\
\text { MULTIDIELECTRIQUES }\end{array}$ \\
\hline & $\begin{array}{c}\text { SPPARAT ION SPECTRALE } \\
\lambda 1 / \lambda 2\end{array}$ & $\begin{array}{c}\text { FILTRE PEROT-FABRY } \\
\text { ( } 3 \text { cavités) }\end{array}$ \\
\hline \multirow{3}{*}{$\begin{array}{c}\text { PERTORMANCES } \\
\text { (valeurs moyennes) }\end{array}$} & PARADIAPHONIE & $\leqslant-30 \mathrm{~dB}$ (opt.) \\
\hline & TELEDIAPHONIE & $\leqslant-50 \mathrm{~dB}$ (opt.) \\
\hline & PERTES D'INSERTION & $\approx-2 \mathrm{~dB}$ \\
\hline
\end{tabular}

\section{Conclusion.}

Le Réseau Local Tout Optique qui élimine les conversions opto-électroniques et qui permet d'utiliser la fibre optique au maximum de ses potentialités par le biais de dispositifs optiques élémentaires comme les miroirs (fonction commutation) ou encore par l'introduction de multiplexeurs démultiplexeurs optiques, est à même de répondre aux spécifications recherchées pour les futurs réseaux multiservices.

Son caractère évolutif et son aptitude à transmettre tous les types de signaux conduisent le CNET à poursuivre les études sur ce sujet avec pour objectifs particuliers la réalisation de matrices optiques de plus grandes capacités : $16 \times 16,32 \times 32$ ou $64 \times 64$ 
et leur implantation dans le cadre d'un réseau local interne.

Mais les possibilités d'utilisation de ces éléments sont nombreuses et peuvent trouver des applications intéressantes dans le cadre de petits réseaux d'entreprises pour l'interconnexion de terminaux, ou d'appareils de saisie de données utilisant des signaux large bande en environnement difficile.

Bibliographie

[1] NTC 80 : Multiservice optical network, TREHEUX, M., LE CoQuIL, E. (CNET).

[2] ECOC 81 : Towards an optical network, TREHEUX, M., LE CoQuil, E. (CNET).

[3] ECOC 82 : Un élément du réseau local tout optique : le concentrateur-distributeur, GENTRIC, A., LE Rouzic, J., MADIGOU, R. (CNET).

[4] ISSS 81 : The optical switching point basis of the future network, LE COQUIL, E. (CNET).

[5] OPTO 82 : Un dispositif de multiplexage optique $a$ 4 canaux, LonNoy, J. (Société SAT, France). 